\title{
First step toward the "fingerprinting" of brain tumors based on synchrotron radiation X-ray fluorescence and multiple discriminant analysis
}

\author{
Magdalena Szczerbowska-Boruchowska • \\ Marek Lankosz • Dariusz Adamek
}

Received: 29 January 2011/ Accepted: 21 June 2011 / Published online: 3 July 2011

(c) SBIC 2011

\begin{abstract}
Synchrotron-radiation-based X-ray fluorescence was applied to the elemental microimaging of neoplastic tissues in cases of various types of brain tumors. The following cases were studied: glioblastoma multiforme, gemistocytic astrocytoma, oligodendroglioma, anaplastic oligodendroglioma, ganglioglioma, fibrillary astrocytoma, and atypical transitional meningioma. Apart from neoplastic tissue, the analysis included areas of tissue apparently without malignant infiltration. The masses per unit area of $\mathrm{P}, \mathrm{S}, \mathrm{Cl}, \mathrm{K}, \mathrm{Ca}, \mathrm{Fe}, \mathrm{Cu}, \mathrm{Zn}, \mathrm{Br}$, and $\mathrm{Rb}$ were used to construct a diagnostic classifier for brain tumors using multiple discriminant analysis. It was found that $\mathrm{S}$, $\mathrm{Cl}, \mathrm{Cu}, \mathrm{Fe}, \mathrm{K}, \mathrm{Br}$, and $\mathrm{Zn}$ are the most significant elements in the general discrimination of tumor type. The highest similarity in elemental composition was between atypical transitional meningioma and fibrillary astrocytoma. The smallest differentiation was between glioblastoma multiforme and oligodendroglioma. The mean percentage of correct classifications, estimated according to the a posteriori probabilities procedure, was $99.9 \%$, whereas the mean prediction ability of $87.6 \%$ was achieved for ten new cases excluded previously from the model construction. The
\end{abstract}

M. Szczerbowska-Boruchowska $(\bowtie) \cdot$ M. Lankosz

AGH University of Science and Technology,

Faculty of Physics and Applied Computer Science,

Al. Mickiewicza 30,

30-059 Kraków, Poland

e-mail: boruchowska@novell.ftj.agh.edu.pl

D. Adamek

Department of Neuropathology,

Chair of Pathomorphology,

Faculty of Medicine,

Jagiellonian University,

Botaniczna 3,

31-503 Kraków, Poland results showed that multiple discriminant analysis based on elemental composition of tissue may be a potentially valuable method assisting differentiation and/or classification of brain tumors.

Keywords Synchrotron radiation - X-ray fluorescence . Brain tumors - Discriminant analysis - Trace elements

\section{Introduction}

The literature published in recent years indicates an essential role played by minor and trace elements in a number of pathological processes [1-3]. They may activate or inhibit enzymatic reactions, participate in binding with other elements or metalloproteins, influence the permeability of cell membranes, etc. $[4,5]$. Therefore, one may assume that selected elements may contribute, directly or indirectly, to the carcinogenic process [5]. Nowadays, there is a growing awareness of, and interest in, studies involving the determination of the elemental composition of normal and malignant tissues [6,7]. Elemental abnormalities, including those related to $\mathrm{Zn}, \mathrm{Cu}, \mathrm{Fe}, \mathrm{K}, \mathrm{Rb}, \mathrm{Mn}, \mathrm{Se}$, and $\mathrm{Ca}$, in various forms of cancer have been reported over the past decade [8-11]. However, the exact role of these and many other elements in carcinogenesis remains unknown.

The process of cancerogenesis may influence normal biochemical reactions leading, among other things, to alterations in the elemental composition of the tissue. Therefore, the levels of chemical elements in neoplastic tissues may differ from those in normal specimens. This feature could be applied in the process of diagnosing cancerous disease, supported by the methods of elemental analysis. Moreover, with respect to the present studies, it was especially interesting if the unique elemental 
composition differentiates not only normal tissue from neoplastic tissue, but also especially among various types of brain tumors. For this purpose two requirements were crucial. Firstly, a multielement analytical technique appropriate for the determination of low concentrations of elements that are typical of brain tissues was required. Moreover, taking into account the inhomogeneous structure of neoplastic tissue (apart from the "intrinsic" heterogeneity of the neoplasm, one has to remember the complex nature of the tumor, which frequently shows admixture of necrotic areas, more or less preserved components of the host tissue, reactive gliosis, and secondary constituents such as macrophages, lymphocytes, monocytes, neutrophils, blood vessels, and calcifications), an analytical method of high spatial resolution, i.e., below $20 \mu \mathrm{m}$, was essential as well. Secondly, a statistical tool that allows differentiation between normal and neoplastic tissues and especially between different types of tumors is of great importance.

Presently, oncology is in need of spatially resolved probes with a chemical sensitivity capable of monitoring biochemical processes and interactions within neoplastic tissues. The recent development of microprobe beamlines of third-generation synchrotron sources enables spatially resolved X-ray fluorescence (XRF) at cellular and subcellular levels. The principle of XRF is based upon the detection of X-rays emitted from sample atoms irradiated with X-rays of higher energy [12]. The energy of the emitted X-rays is characteristic of the excited element, thus enabling the identification of the content of the elements present in the sample. Synchrotron-radiation-based XRF (SR-XRF) microprobe analysis is a multielemental analytical technique which enables the simultaneous microimaging of chemical elements at trace concentrations. For this reason, XRF seems to be a promising tool for the investigation of cancerogenesis. SR-XRF has been used in a number of applications in biology and medicine [13, 14]. A comprehensive overview of recent applications of XRF microscopy in biology and medicine was presented by Paunesku et al. [15]. The field of applications of SR-XRF includes research on cancer chemotherapy of inorganic compounds, physiology of trace elements, and metal neurotoxicity [16-19]. Since alterations in homeostasis of trace elements, in particular $\mathrm{Cu}, \mathrm{Fe}$, and $\mathrm{Zn}$, may play an important role in selected neurodegenerative disorders, including Alzheimer's disease, amyotrophic lateral sclerosis, and Parkinson's disease, the SR-XRF technique was used to visualize the metal ion distribution in brain tissue or nerve cells [20-26]. SR-XRF was also applied with respect to different tumors [13, 27-30]. The elemental abnormalities in neoplastic tissues have also been investigated.

As mentioned previously, statistical techniques for the analysis and interpretation of the experimental data are necessary. Multiple discriminant analysis (MDA), being an extension of discriminant analysis, is a powerful technique for examining differences between two or more groups of objects (in this case neoplastic tissues) with respect to several variables simultaneously (in this case tissue element levels) [31-34]. Generally, the two main goals of discriminant analysis are (1) to build a statistically significant model that enables group differentiation based on predictor variables and (2) to classify the dependent variable using the model calculated. Generally, discriminant analysis is a well-known technique for dimension reduction, feature extraction, and case classification. MDA has been used widely in many applications, including various aspects of oncogenesis [13, 32-34]. Discriminant analysis was used for distinguishing different types of brain tumors on the basis of magnetic resonance spectra [35]. The potential application of discriminant analysis and infrared spectroscopic imaging as a diagnostic method in astrocytic gliomas was reported by Krafft et al. [36]. Small-angle $\mathrm{X}$-ray scattering patterns of benign and malignant brain tumor tissue were examined using flexible discriminant analysis [37]. The features of brain tumors related to their elemental composition had not been previously studied.

Establishing the pattern of the distribution of elements characteristic of a particular brain tumor is by itself a tempting goal, although even if achievable, it could neither be easily explained nor interpreted in the context of the metabolism or mechanisms of carcinogenesis. Nevertheless, a sort of "elemental fingerprinting" of brain tumors could provide a very useful tool assisting the process of diagnosing tumors in difficult or disputable cases. The aim of this study was to see if the MDA of elements commonly found in brain tumor tissues can be used to differentiate neoplastic samples according to their histopathological classifications.

\section{Materials and methods}

\section{Sample preparation}

The samples for biochemical microimaging were taken intraoperatively from brain tumors of different types and various grades of malignancy. The samples were diagnosed histopathologically in the Department of Neuropathology of Jagiellonian University Medical College in Kraków. The samples from the following tumor types were investigated using SR-XRF (with the grade of tumor malignancy according to the latest WHO classification [38] given in parentheses): glioblastoma multiforme (IV), gemistocytic astrocytoma (II), oligodendroglioma (II), anaplastic oligodendroglioma (III), ganglioglioma (I), fibrillary astrocytoma (II), and atypical transitional meningioma (II). In 
Table 1 Specification of the brain tumors used in the analysis

\begin{tabular}{lll}
\hline Tumor type & WHO grade & Number of cases \\
\hline GM & IV & 11 \\
AG & II & 2 \\
O & II & 4 \\
OA & III & 2 \\
G & I & 1 \\
AF & II & 1 \\
M & II & 1 \\
C & & 1
\end{tabular}

$\overline{G M}$ glioblastoma multiforme, $A G$ gemistocytic astrocytoma, $O$ oligodendroglioma, $O A$ anaplastic oligodendroglioma, $G$ ganglioglioma, $A F$ fibrillary astrocytoma, $M$ atypical transitional maningioma, $C$ control

addition to the neoplastic tissues, the analysis also included an area of tissue apparently without malignant infiltration (serving as a control group). The control sample was removed intraoperatively together with the specimen of anaplastic oligodendroglioma. More detailed information about the cases investigated is given in Table 1.

For each tumor studied, samples taken for standard histopathological intraoperational examination were cryosectioned at $5 \mu \mathrm{m}$ for routine hematoxylin-eosin (HE) staining and at $20 \mu \mathrm{m}$ for spectroscopic elemental microimaging analysis. The slices destined for elemental analysis were mounted immediately onto Ultralene foil of $4-\mu \mathrm{m}$ thickness suspended in a Plexiglas holder and finally freeze-dried at $-80{ }^{\circ} \mathrm{C}$. Additional samples of tissue, routinely embedded in paraffin, were used for definite histopathological diagnosis, i.e., tumor type and grade, using HE and other staining methods, if necessary including also immunohistochemistry (most frequently with antibodies against glial fibrillary acidic protein, cytokeratins, epithelial membrane antigen, vimentin, CD34, and others, accordingly to the specific requirements of differential diagnosis in every particular case).

All samples were examined microscopically to select the appropriate regions for elemental investigation, i.e., to exclude from the analysis any artifacts, large necrotic areas, blood vessels, extensive calcifications, etc. In other words, in every case efforts were undertaken to select the most representative tumor tissue for elemental analysis. Moreover, microscopic histological examination of corresponding sections was used to choose the region designed for elemental analysis. An example of white-light microscope images of an HE-stained tissue section and the corresponding unstained slice selected for the elemental mapping is presented in Fig. 1 .

\section{Measurements conditions}

The SR-XRF measurements were performed at the bending magnet beamline $\mathrm{L}$ at Hamburger Synchrotrostrahlunglabor (HASYLAB; Hamburg, Germany) [39]. The primary X-ray energy defined with the use of a multilayer $\mathrm{Ni} / \mathrm{C}$ monochromator was set to $17 \mathrm{keV}$. To reduce the beam size, a polycapillary half-lens for monochromatic applications which require maximum flux was used. The beam was focused to a size of $15 \mu \mathrm{m}$ in diameter. The typical flux of the incoming radiation, measured before the sample, was about $8 \times 10^{10}$ photons $/ \mathrm{s}$. The tissue samples were positioned at an angle of $45^{\circ}$ with respect to the incident
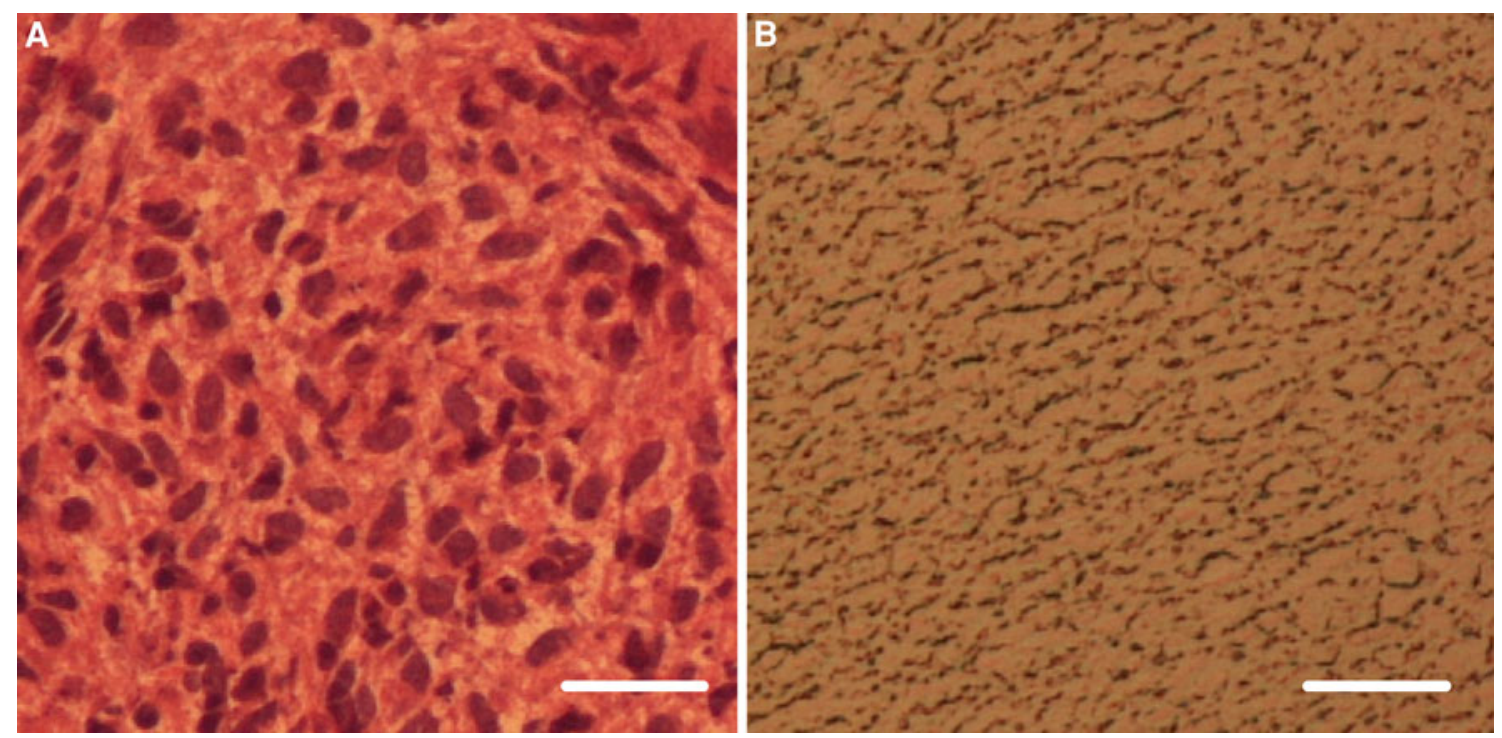

Fig. 1 Glioblastoma multiforme-microscopic view of the sample region selected for elemental analysis: a stained with hematoxylin-eosin; b unstained tissue section. Scale bars $50 \mu \mathrm{m}$ 
beam. The sample areas were scanned with a step size of $15 \mu \mathrm{m}$ both horizontally and vertically. The size of the area of scanning depended on the tissue structure but was typically 300-500 $\mu \mathrm{m}$ in one direction. The acquisition time was $10 \mathrm{~s}$ for each measurement point. The measurements were conducted in air. The characteristic X-ray lines were measured by a Vortex silicon drift detector from SII Nano Technology USA. The sample-detector distance was set at about $15 \mathrm{~mm}$. To perform elemental mapping of the tissue, the position of the sample was changed with respect to the incident beam using a computer-controlled stepper motor allowing micrometer movements. Spectrometer calibration was performed on the basis of measurements of standard reference materials (NIST SRM 1832 and NIST SRM 1833).

Spectral analysis

Evaluation of the XRF spectra was performed using the AXIL-QXAS software package freely distributed by the IAEA [40]. It is a routinely used computer program designed specifically for qualitative and quantitative analysis of XRF spectra. With use of the AXIL module, a declared group of X-ray lines (e.g., the $\mathrm{K}$ series) of a given element is treated by the fitting procedure as a single entity. The net peak area is determined for each group during the spectrum fitting (separately for each band). Lines within a group have fixed intensity ratios during the fit. The X-ray energies and the relative intensities are read from the appropriate library file. In our study, to avoid any problems related to the estimation of the peak areas of small or strongly overlapping peaks, all the lines of one element were specified in the spectral analysis as one group instead of entering elemental X-ray lines separately. Moreover, during the spectrum fitting using the AXIL module, $\mathrm{K}$-series peaks were approximated by Gaussian functions. The net peak areas of $\mathrm{K} \alpha$ lines were used in further quantitative evaluation. The calculated intensities of elemental X-ray lines were normalized to the value of the incident photon flux.

\section{Statistical analysis}

One of the multivariate methods, i.e., MDA, was applied to the grouping and classification of brain tissue samples based on their elemental content. In this study the main goals of the statistical analysis were (1) to accomplish data reduction and simplification, (2) to determine which elements contribute most to the discrimination between groups, and (3) to construct a diagnostic classifier for brain tumors using elemental composition.

Mathematically, the aim of discriminant analysis was to find a set of linear combinations of the variables (called discriminant functions) whose values were as close as possible within groups (tumor types) and as far apart as possible between groups [41]. The discriminant functions were generated from a sample of cases for which group membership was known. The functions were then applied to new cases with measurements on the same set of variables, but unknown group membership. The most common test for the statistical significance of the discriminant function is based on the residual discrimination in the system prior to deriving that function. The most appropriate formula in this context is Wilks's lambda [31], which was applied in this work. The detailed theoretical basis of the discriminant analysis technique was presented elsewhere [41].

The statistical package STATISTICA 7.1 from StatSoft [42] was used for discriminant analysis calculations.

\section{Results}

A typical XRF spectrum from brain tumor tissue excited by means of synchrotron radiation is shown in Fig. 2. The SR$\mathrm{XRF}$ technique revealed that elements such as $\mathrm{P}, \mathrm{S}, \mathrm{Cl}, \mathrm{K}$, $\mathrm{Ca}, \mathrm{Fe}, \mathrm{Cu}, \mathrm{Zn}, \mathrm{Br}$, and $\mathrm{Rb}$ were present in all the neoplastic tissues analyzed. Moreover, in selected cases Se and $\mathrm{Sr}$ were also detected. However, the contents of these elements in neoplastic tissues are relatively low. Therefore, under the applied measurement conditions, Se and Sr were not determined in all the cases studied as part of the experiment. Consequently, these elements were excluded from further statistical analysis.

SRM 1832 and SRM 1833 were used as calibration standards in the calculation of masses per unit area of the elements in this study. For calibration of sensitivity versus atomic number, the fitting curve was constructed on the basis of the certified values of the masses per unit area of

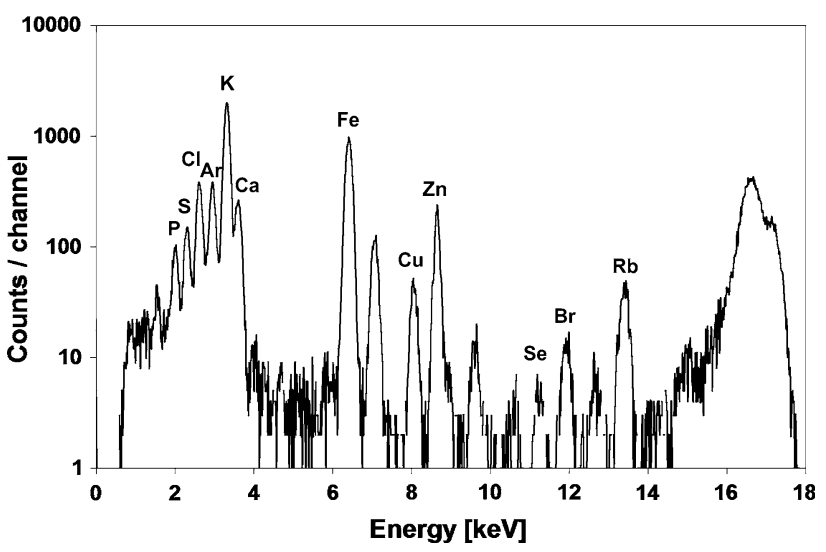

Fig. 2 A typical spectrum of brain glioma tissue excited by synchrotron radiation (beam energy $17 \mathrm{keV}$, acquisition time $10 \mathrm{~s}$ per point). X-ray fluorescence $\mathrm{K} \alpha$ lines of the elements are shown) 
elements in the standard reference materials. The sensitivity for each analyzed element $i$ was calculated using the following expression:

$S_{i}=Y_{\mathrm{s}} /\left(F_{\mathrm{s}} M_{\mathrm{s}}\right)$,

where $Y_{\mathrm{s}}$ is the net peak area of the measured element for the standard sample (SRM 1832 or SRM 1833), $F_{\mathrm{s}}$ is the incident photon flux in the measurement of the reference material, and $M_{\mathrm{s}}$ is the mass per unit area of the measured element in the standard sample.

On the basis of the spectral data from each point of the scanned areas of the samples, the masses per unit area of elements were determined according to the following formula:

$M=Y_{\mathrm{t}} /\left(F_{\mathrm{t}} S_{i}\right)$,

where $Y_{\mathrm{t}}$ is the net peak area of the measured element in the tissue sample, $F_{\mathrm{t}}$ is the incident photon flux in the measurement of the tissue sample, and $S_{i}$ is sensitivity for measured element $i$.

The calculated values of the masses per unit area of elements were applied to obtain two-dimensional maps of elemental distribution. This allowed not only the recognition of the topography of elements, but also avoidance of any elemental artifacts that would further falsify the analysis. For each case the representative area was selected from the whole scanned region of the sample. The distribution of elements in such a representative area of a glioblastoma multiforme sample is presented in Fig. 3 . Comparable areas were selected for other samples.

The masses per unit area of elements from the representative region of each sample were used for further statistical analysis. Typically, the data came from 101 points in each case. Statistical analysis was performed only for these elements that were detected in all the samples studied, i.e., for $\mathrm{P}, \mathrm{S}, \mathrm{Cl}, \mathrm{K}, \mathrm{Ca}, \mathrm{Fe}, \mathrm{Cu}, \mathrm{Zn}, \mathrm{Br}$, and $\mathrm{Rb}$. The mean values of the masses per unit area of elements in the analyzed sample areas are shown in Fig. 4.

Prior to statistical analysis, the values of the masses per unit area of elements were transformed to log base 10. One reason for this was to make the distribution more symmetric and Gaussian-like, which was not fulfilled for raw data in all cases studied. Moreover, logarithmic transformation was applied to make the variances of the variables in the analyzed groups more homogeneous in a nonstandardized multivariate statistical analysis. The transformation of data to $\log$ base 10 before a multivariate statistical method is a widely used procedure [43].

Discriminant function analysis was divided into two steps: (1) detecting the variables that allow discrimination between different tumor types and (2) classifying cases into groups related to histopathological recognition. As already mentioned, the main criterion for determining the elements contributing significantly to a particular grouping of the analyzed cases was the minimization of Wilks's lambda. Discriminant functions for distinguishing between tumor
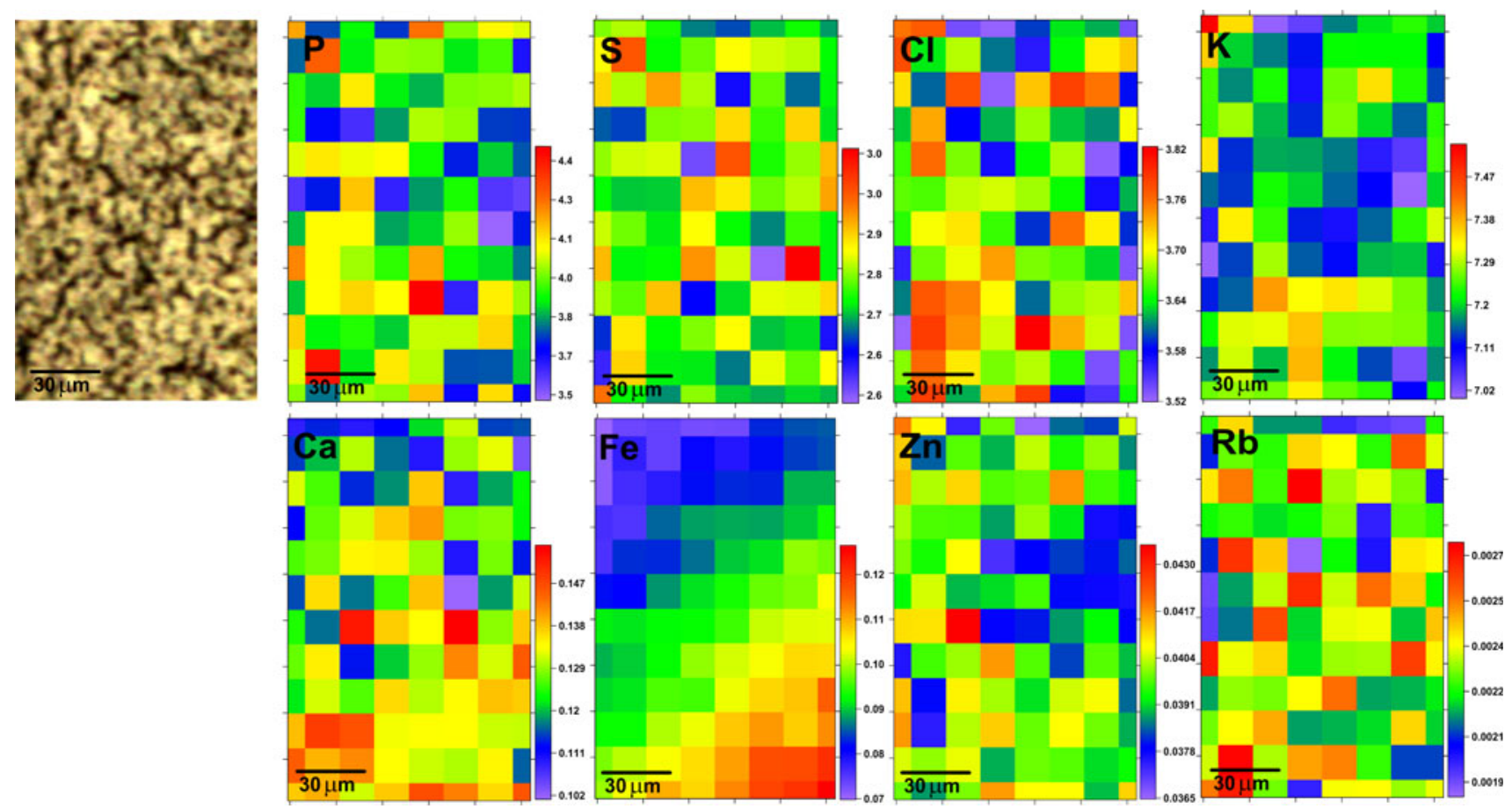

Fig. 3 Distribution of selected elements in a glioblastoma multiforme tissue section in comparison with the visible image of the scanned area of the tissue. Data are presented in micrograms per square centimeter 

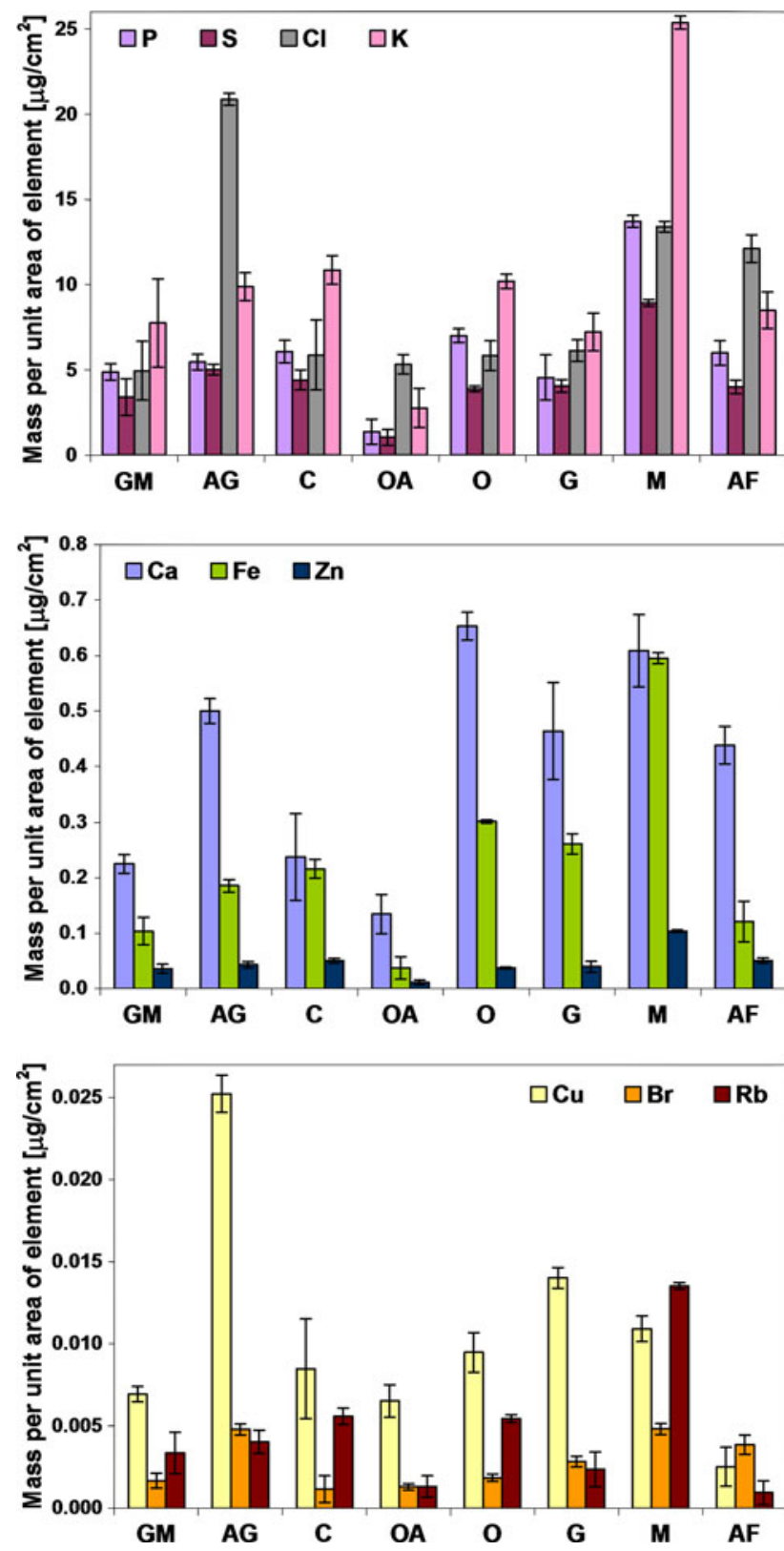

Fig. 4 Mean values of masses per unit area of elements $\left(\mu \mathrm{g} / \mathrm{cm}^{2}\right)$ detected in brain tumors and control samples. Error bars represent standard deviations of mean values at the $95 \%$ confidence interval. $G M$ glioblastoma multiforme, $A G$ gemistocytic astrocytoma, $C$ control, $O A$ anaplastic oligodendroglioma, $O$ oligodendroglioma, $G$ ganglioglioma, $M$ atypical transitional maningioma, $A F$ fibrillary astrocytoma

types, including noncancerous tissue, were calculated by the forward stepwise procedure.

In the statistical analysis, the samples were discriminated into groups on the basis of their histopathological diagnosis (according to the data given in Table 1). All the elements detected (primary variables) were statistically significant in the discrimination and were included in the model built. The parameters describing the significance of
Table 2 Parameters describing the significance of the primary variables (elements) for the model

\begin{tabular}{llcl}
\hline Element & Partial Wilks's $\Lambda$ & Fisher's $F$ statistics & $p$ \\
\hline $\mathrm{S}$ & 0.076 & 2,110 & $\ll 0.05$ \\
$\mathrm{Cl}$ & 0.14 & 1,120 & $\ll 0.05$ \\
$\mathrm{Cu}$ & 0.19 & 750 & $\ll 0.05$ \\
$\mathrm{Fe}$ & 0.19 & 750 & $\ll 0.05$ \\
$\mathrm{~K}$ & 0.20 & 690 & $\ll 0.05$ \\
$\mathrm{Br}$ & 0.25 & 530 & $\ll 0.05$ \\
$\mathrm{Zn}$ & 0.29 & 430 & $\ll 0.05$ \\
$\mathrm{P}$ & 0.49 & 190 & $\ll 0.05$ \\
$\mathrm{Ca}$ & 0.56 & 140 & $\ll 0.05$ \\
$\mathrm{Rb}$ & 0.98 & 4 & $\ll 0.05$ \\
\hline
\end{tabular}

the primary variables for the model, including partial Wilks's lambda, the $F$ test of partial Wilks's lambda, and its $p$ values, are summarized in Table 2. It was found that $\mathrm{S}, \mathrm{Cl}, \mathrm{Cu}, \mathrm{Fe}, \mathrm{K}, \mathrm{Br}$, and $\mathrm{Zn}$ are the most significant elements in the general discrimination of tumor type.

The canonical variables and the discriminant functions can be expressed as a linear combination of the masses per unit area of elements included in the particular model. The statistical analysis, whose goal was to differentiate the samples on the basis of their diagnosis, allowed the identification of eight discriminant functions. All of them were statistically significant. By comparing their eigenvalues, which reflect the ratio of importance to the group discrimination, we selected two of them with the highest eigenvalues for further analysis. The equations of the canonical roots chosen are as follows:

$$
\begin{aligned}
L_{1}= & 0.754 \times \mathrm{P}+2.33 \times \mathrm{S}-2.12 \times \mathrm{Cl}-3.37 \\
& \times \mathrm{K}+0.82 \times \mathrm{Ca}-0.930 \times \mathrm{Fe}+0.442 \times \mathrm{Cu} \\
& -0.601 \times \mathrm{Zn}+0.681 \times \mathrm{Br}-0.074 \times \mathrm{Rb}, \\
L_{2}= & 1.85 \times \mathrm{P}-1.96 \times \mathrm{S}+0.506 \times \mathrm{Cl}+0.632 \times \mathrm{K} \\
& -0.115 \times \mathrm{Ca}+0.544 \times \mathrm{Fe}-0.879 \times \mathrm{Cu}-0.991 \\
& \times \mathrm{Zn}-0.075 \times \mathrm{Br}+0.020 \times \mathrm{Rb} .
\end{aligned}
$$

The chemical symbols of elements represent discriminating variables, i.e., masses per unit area of elements. As can be seen, $\mathrm{K}, \mathrm{S}$, and $\mathrm{Cl}$ are the most significant elements in the first discrimination function, whereas in the second one they are $\mathrm{S}$ and $\mathrm{P}$.

An examination of the group discrimination was performed on the basis of a simple scatter plot between two discrimination variables. The distribution of the samples in the plane of the two calculated discriminant functions is illustrated in Fig. 5. The scatter plot shows that discriminant function 1 discriminates mostly between fibrillary astrocytoma and other analyzed cases (the highest distance in the horizontal direction). In the vertical direction 


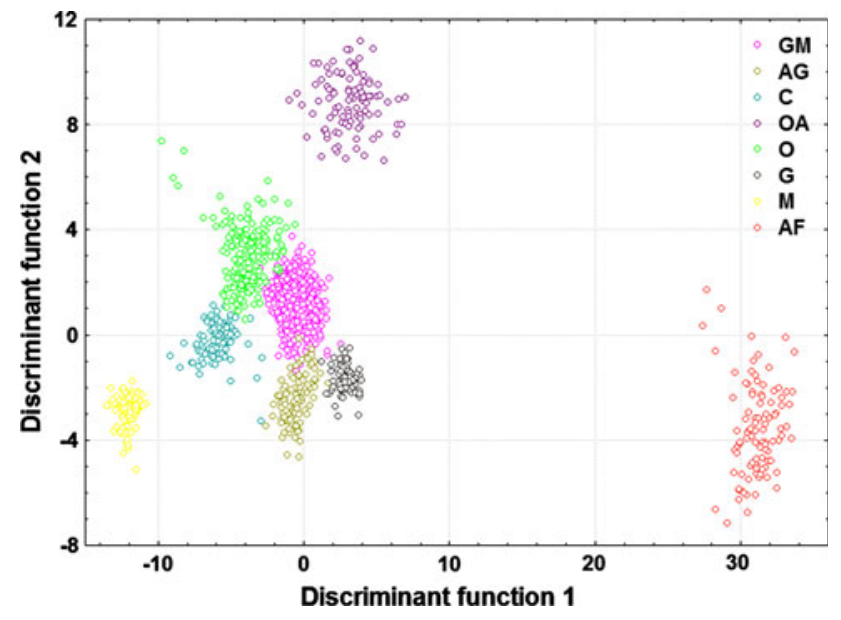

Fig. 5 The scatter plot of observations in the space of discriminant variables for different types of brain tumors and the control group

Table 3 The mean values of the calculated canonical functions across groups

\begin{tabular}{llc}
\hline Tumor type & \multicolumn{2}{l}{ Discriminant function } \\
\cline { 2 - 3 } & 1 & 2 \\
\hline GM & -0.48 & 1.2 \\
AG & -0.54 & -2.5 \\
C & -5.8 & -0.22 \\
OA & 3.2 & 8.8 \\
O & -3.8 & 3.0 \\
G & 2.3 & -1.7 \\
M & -12 & -2.8 \\
AF & 31 & -3.7 \\
\hline
\end{tabular}

(discriminant function 2), one slight trend appears: anaplastic oligodendroglioma points are above the clusters for other tumors. These findings are in accordance with the calculated means for canonical variables (see Table 3).

To measure differentiation between examined groups in the space of discriminant variables, the squared Mahalanobis distances were calculated. The results are presented in Table 4. All the calculated Mahalanobis distances were statistically significant $(p \ll 0.05)$, which confirms the significance of the differences between the analyzed groups. It was found that the largest Mahalonobis distance, which reflects the largest difference in elemental composition, was between the centroids of atypical transitional meningioma and fibrillary astrocytoma. The smallest differentiation, taking into account the elemental composition of neoplastic tissues, was between glioblastoma multiforme and oligodendroglioma.

The utility of the calculated canonical roots was examined via their ability to correctly classify each data point to their a priori groups. Classification functions were derived to achieve this purpose. The classification matrix obtained is presented in Table 5. The results are shown for the cases used in the construction of the model as well as for new cases. The degree of success of the classification of each group was summarized by the number of cases correctly classified and misclassified. For each group, two results of the classification of the samples are shown: the diagnosis given by a histopathologist and judgment formed by taking into account the calculated model. The mean percentage of correct predictions estimated according to the a posteriori probabilities procedure was $99.93 \%$. In all cases almost $100 \%$ agreement with histopathological diagnosis was achieved.

Additionally, the leave-one-out method was used as a cross-validation procedure to evaluate the classification efficiency of the model constructed previously. For this purpose ten new cases were used. The following brain gliomas were used: oligodendroglioma (two cases), anaplastic oligodendroglioma (one case), gemistocytic astrocytoma (one case), glioblastoma multiforme (six cases). As previously, for each sample the elemental mass fractions from 101 points of a representative area were taken for the validation. The mean percentage of correct predictions obtained for the new cases of brain gliomas that were not used in the construction of the model was $87.6 \%$. The lowest prediction ability (85.8\%) was achieved for glioblastoma multiforme. In this case, 520 observations of 606 points analyzed were classified according to the histopathological diagnosis. The highest score $(99 \%$ of correct
Table 4 Squared Mahalanobis distances between group centroids

The numbers in bold are the smallest and largest distances

\begin{tabular}{lrrrrrrrr}
\hline & GM & \multicolumn{1}{c}{ AG } & \multicolumn{1}{c}{ C } & OA & O & G & M & AF \\
\hline GM & - & 110 & 50 & 140 & $\mathbf{2 0}$ & 50 & 180 & 1,010 \\
AG & 110 & - & 190 & 160 & 150 & 110 & 220 & 1,070 \\
C & 50 & 190 & - & 260 & 45 & 140 & 110 & 1,370 \\
OA & 140 & 160 & 260 & - & 170 & 190 & 470 & 990 \\
O & $\mathbf{2 0}$ & 150 & 45 & 170 & - & 85 & 120 & 1,250 \\
G & 50 & 110 & 140 & 190 & 85 & - & 280 & 820 \\
M & 180 & 220 & 110 & 470 & 120 & 280 & - & $\mathbf{1 , 8 5 0}$ \\
AF & 1,010 & 1,070 & 1,370 & 990 & 1,250 & 820 & $\mathbf{1 , 8 5 0}$ & - \\
\hline
\end{tabular}


Table 5 Classification results for brain tumor tissue discrimination

\begin{tabular}{|c|c|c|c|c|c|c|c|c|c|}
\hline \multirow[t]{2}{*}{ Recognition } & \multirow[t]{2}{*}{ Correctly classified $(\%)$} & \multicolumn{8}{|c|}{ Predicted group membership } \\
\hline & & GM & AG & $\mathrm{C}$ & $\mathrm{OA}$ & $\mathrm{O}$ & G & M & $\mathrm{AF}$ \\
\hline \multicolumn{10}{|c|}{ Classification of the cases used to the model construction } \\
\hline GM & 99.8 & 504 & 0 & 0 & 0 & 0 & 1 & 0 & 0 \\
\hline AG & 100 & 0 & 101 & 0 & 0 & 0 & 0 & 0 & 0 \\
\hline $\mathrm{C}$ & 100 & 0 & 0 & 101 & 0 & 0 & 0 & 0 & 0 \\
\hline OA & 100 & 0 & 0 & 0 & 101 & 0 & 0 & 0 & 0 \\
\hline $\mathrm{O}$ & 100 & 0 & 0 & 0 & 0 & 202 & 0 & 0 & 0 \\
\hline G & 100 & 0 & 0 & 0 & 0 & 0 & 101 & 0 & 0 \\
\hline M & 100 & 0 & 0 & 0 & 0 & 0 & 0 & 101 & 0 \\
\hline $\mathrm{AF}$ & 100 & 0 & 0 & 0 & 0 & 0 & 0 & 0 & 101 \\
\hline Total & 99.93 & 504 & 101 & 101 & 101 & 202 & 102 & 101 & 101 \\
\hline \multicolumn{10}{|c|}{ Classification of the new cases } \\
\hline GM & 85.8 & 520 & 0 & 23 & 0 & 0 & 58 & 3 & 2 \\
\hline AG & 88.1 & 0 & 89 & 0 & 0 & 0 & 0 & 12 & 0 \\
\hline $\mathrm{OA}$ & 99.0 & 0 & 0 & 0 & 100 & 1 & 0 & 0 & 0 \\
\hline $\mathrm{O}$ & 87.1 & 22 & 0 & 0 & 0 & 176 & 2 & 2 & 0 \\
\hline Total & 87.6 & 542 & 89 & 23 & 100 & 177 & 60 & 17 & 2 \\
\hline
\end{tabular}

classifications) was achieved for anaplastic oligodendroglioma. For this kind of tumor only one observation was classified as oligodendroglioma.

\section{Discussion}

As shown in Fig. 2, the SR-XRF technique enabled the determination of a wide range of elements between $\mathrm{P}$ and $\mathrm{Rb}$. Generally, the simultaneous trace element sensitivity and micrometer spatial resolution makes the technique indispensable with respect to the quantitative mapping of trace element distributions in tissue samples at single-cell level. In our studies, the technique allowed the identification of a two-dimensional distribution of elements on a microscale. As illustrated in Fig. 3, the representative areas selected for each sample were characterized by quite a homogeneous distribution of elements. The comparison of the microscopic image of the scanned area of tissues with the XRF maps showed that the distribution of elements reflects apparently uniform morphology of the tissue. However, as one can see, the distribution of $\mathrm{Fe}$ is unlike that of any other element. The XRF map of Fe clearly shows a concentration gradient over the entire area. Therefore, it may raise concerns that the Fe mass per unit area in such a selected part of the section is not really representative. It is worth pointing out that this particular distribution of $\mathrm{Fe}$ in the case presented was not typical of other samples. However, in such questionable cases the use of data from a larger sample area is recommended. The results shown in Fig. 3 relate to the case of glioblastoma multiforme. Since in our study four additional samples representing this tumor type were included, in further statistical analysis we decided not to extend the data set. Moreover, the abnormal distribution was found only for one element. That is why the selected area was finally regarded as representative. In spite of the morphological homogeneity of the tissue seen under the microscope and a homogenous distribution of most of the analyzed elements, some topographic and quantitative anomalies for a single element may occur.

MDA allowed for the discovery that $\mathrm{S}, \mathrm{Cl}, \mathrm{Cu}, \mathrm{Fe}, \mathrm{K}$, $\mathrm{Br}$, and $\mathrm{Zn}$ are the elements of the highest significance in the general discrimination of tumor type. This suggests that the contents of these elements and particularly their relative ratios in tissues may constitute a source of the unique elemental fingerprint of different types of brain tumors. It should be emphasized that the possible role of these elements in oncogenetic processes has been previously reported [8-11].

Moreover, the results obtained showed that the elemental composition of a relatively small fragment of homogeneous tissue represents satisfactorily the biochemical "signature" of cancer. On the basis of the element levels determined in such a small sample by means of the SR-XRF technique and MDA it was possible to differentiate various types of brain tumors (see Fig. 5). The scatter plot of two discrimination variables showed eight clearly separated classes corresponding to the histopathological diagnosis of brain tumors and the control sample. As can be seen, some tumor types are represented by only one case, which could be questionable from a statistical point of 
view. However, as shown in Fig. 5, all the data representing five cases of glioblastoma multiforme are located in one centroid separated from the groups of other tumor types. It is worth emphasizing that the model of discriminant functions obtained in this work did not differentiate the samples representing five various cases of glioblastoma multiforme. The same result was observed for two samples of oligodendroglioma. Moreover, to validate the proposed method and verify its usefulness in predictive assumptions, the new cases were included in the classification procedure.

SR-XRF analysis coupled with MDA allowed the classification of different types of brain tumors into separate groups and also even the use of statistical calculation to classify the unknown cases. It is worth emphasizing that in this study very high predictive accuracies of MDA were achieved. As mentioned earlier, the mean percentage of correct predictions estimated according to the a posteriori probability procedure was $99.93 \%$. It should be emphasized that the prediction ability of about $88 \%$ was achieved in the classification of new cases of brain tumors which had been previously excluded from the construction of the model. This suggests that the elemental fingerprinting created may be a very useful tool in assisting the process of histopathological diagnosis of tumors, especially in difficult or disputable cases.

One has to remember that conceptually the process of histopathological diagnosis of tumors is a complex one, and is based on the verbatim interpretation of the pictures (the microscopic ones) of tissue samples. In diagnosing, histopathologists (or neuropathologists) use their expertise, and knowledge gained from literature, and, especially in difficult cases or when the tissue material is limited (e.g., stereotactic biopsy), also other sources of data, i.e., a clinical, radiological (neuroimaging) picture in a given case [44].

In other words, nothing is directly given in a microscopic picture of the tumor sample. This may sometimes result in discrepancies in diagnosis between neuropathologists, even seemingly the best ones, and probably oligodendroglial tumors are among the most susceptible with regard to the differences in neuropathological interpretation ("misinterpretation" in this context is rather improper) [45]. What is more, the very basic issue of differentiation between reactive, i.e. non-neoplastic glial reaction (so called gliosis or astrogliosis) and low grade astrocytoma has not been definitely solved. Astrogliosis is unspecific glial reaction that accompanies many pathological lesions like abscesses, other inflammatory conditions, prion diseases, demyelination, and also tumors themselves. In some instances reactive gliosis may be even misinterpreted as low grade astrocytoma [46]. In difficult cases the "elemental profile," if accessible, could serve as a helpful accessory diagnostic tool.
We are aware that the interpretation of the distribution of elements within tumor tissue in the context of, say, metabolism or molecular content, let alone in the context of the attempt to couple it somehow with the concepts of pathogenesis, may be extremely difficult. Moreover, the application of the synchrotron-radiation-based technique as a diagnostic tool is highly impractical. This is mainly due to the very limited measurement time in the synchrotron facilities, which are usually not readily available for routine use. In this study, this excellent X-ray source was applied. However, as already mentioned, an X-ray beam of about $20-\mu \mathrm{m}$ diameter is appropriate for the studies presented here. Therefore, less precise laboratory microbeam XRF spectrometers equipped with a high-power fine-focus $\mathrm{X}$-ray tube (e.g., with a rotating anode) and X-ray optics (polycapillaries or Kirkpatrick-Baez mirrors) that maintain a high flux of incoming radiation at micrometrer spatial resolution could be applied for such analysis.

An important issue relating to the application of an $\mathrm{XRF}$ microprobe as a diagnostic tool is optimal spatial resolution for such analysis. A complex and inhomogeneous morphology of neoplastic tissues imposes limitations on the incident beam size. The use of a relatively large X-ray beam size, i.e., about 50-100 $\mu \mathrm{m}$, may falsify the analysis because the measured spot may include both the representative neoplastic tissue and various unwanted inclusions which often occur in neoplastic tissues. On the other hand, the high spatial resolution, i.e., below $5 \mu \mathrm{m}$, which allows the determination of the elemental composition of the specimen at a subcellular level, seems to be useless in this study. In fact, analysis based exclusively on cancer cells gives information only about the main tumor components. However, the precise location of a single cell, when using in the experiment the unstained tissue section, is extremely difficult in practice. Therefore, the XRF mapping of specimens at a spatial resolution comparable to cancer cell dimensions, i.e., about $10-20 \mu \mathrm{m}$, seems to be reasonable. Therefore, laboratory X-ray sources of high intensity could be potentially used in place of a synchrotron.

A well-established "elemental signature" of a tumor, if (hopefully) achievable, could turn out to be truly practical and available for clinical use. Certainly nobody could imagine replacing routine histopathological diagnostic methods by elemental fingerprinting as is done with magnetic resonance spectroscopy, which is broadly used but only as an auxiliary method in diagnosing central nervous system tumors. It is not easy to speculate on the meaning of our findings from the biochemical perspective, however. The MDA based on the elemental composition of tissue may be a potentially valuable method in assisting the differentiation and/or classification (diagnosis) of brain tumors. 
Acknowledgments We acknowledge HASYLAB/DESY for the provision of synchrotron radiation facilities. We thank Karen Appel, Anna Smykla, and Mateusz Czyzycki for their assistance in the experiment as well as Zdzislaw Stegowski and Joanna Jaskiewicz for data preprocessing. This work was supported by the Ministry of Science and High Education, Warsaw, Poland, and the following grants: DESY/304/2006 (Ministry of Science and High Education, Warsaw, Poland, 2006-2009), N N518 377537 (Ministry of Science and High Education, Warsaw, Poland), and the European Community's Seventh Framework Programme (FP7/2007-2013) under grant agreement no. 226716. The study was approved by Jagiellonian University Bioethical Committee (KBET/101/B/2010).

\section{References}

1. Valko M, Rhodes CJ, Moncol J, Izakovic M, Mazur M (2006) Chem Biol Interact 160:1-40

2. Valko M, Morris H, Cronin MT (2005) Curr Med Chem 12:1161-1208

3. Jomova K, Vondrakova D, Lawson M, Valko M (2010) Mol Cell Biochem 345:91-104

4. Vural H, Uzun K, Uz E, Koçyigit A, Cigli A, Akyol O (2000) J Trace Elem Med Biol 14:88-91

5. Taysi S, Polat F, Gul M, Sari RA, Bakan E (2002) Rheumatol Int 21:200-204

6. Sky-Peck HH (1986) Clin Physiol Biochem 4:99-111

7. Millos J, Costas-Rodríguez M, Lavilla I, Bendicho C (2008) Anal Chim Acta 622:77-84

8. Al-Ebraheem A, Farquharson MJ, Ryan E (2009) Appl Radiat Isot 67:470-474

9. Gurusamy KS, Farquharson MJ, Craig C, Davidson BR (2008) Biometals 21:373-378

10. Lavilla I, Costas M, Miguel PS, Millos J, Bendicho C (2009) Biometals 22:863-875

11. Piacenti da Silvaa M, Araújo OL, Zucchib D, Ribeiro-Silva A, Poletti ME (2009) Spectrochem Acta B 64:587-592

12. Adams F, Janssens K, Snigirev A (1998) J Anal At Spectrom 13:319-331

13. Banaś K, Jasiński A, Banaś AM, Gajda M, Dyduch G, Pawlicki B, Kwiatek WM (2007) Anal Chem 79:6670-6674

14. Huang YY, Lu JX, He RG, Zhao LM, Wang ZG, He W, Zhang YX (2001) Nucl Instrum Methods Phys Res A 467-468:1301-1304

15. Paunesku T, Vogt S, Maser J, Lai B, Woloschak G (2006) J Cell Biochem 99:1489-1502

16. Ilinski P, Lai B, Cai Z, Yun W, Legnini D, Talarico T, Cholewa M, Webster LK, Deacon GB, Rainone S, Phillips DR, Stampfl AP (2003) Cancer Res 63:1776-1779

17. Finney L, Mandava S, Ursos L, Zhang W, Rodi D, Vogt S, Legnini D, Maser J, Ikpatt F, Olopade OI, Glesne D (2007) Proc Natl Acad Sci USA 104:2247-2252

18. Duong TT, Witting PK, Antao ST, Parry SN, Kennerson M, Lai B, Vogt S, Lay PA, Harris HH (2009) J Neurochem 108:11431154

19. Kehr S, Malinouski M, Finney L, Vogt S, Labunskyy VM, Kasaikina MV, Carlson BA, Zhou Y, Hatfield DL, Gladyshev VN (2009) J Mol Biol 389:808-818
20. Szczerbowska-Boruchowska M, Chwiej J, Lankosz M, Adamek D, Wojcik S, Krygowska-Wajs A, Tomik B, Bohic S, Susini J, Simionovici A, Dumas P, Kastyak M (2005) X-Ray Spectrom 34:514-520

21. Ortega R, Cloetens P, Devès G, Carmona A, Bohic S (2007) PLoS ONE 2(9):e925. doi:10.1371/journal.pone.0000925

22. Tomik B, Chwiej J, Szczerbowska-Boruchowska M, Lankosz M, Wójcik S, Adamek D, Falkenberg G, Bohic S, Simionovici A, Stegowski Z, Szczudlik A (2006) Neurochem Res 31:321-331

23. Yoshida S, Ide-Ektessabi A, Fujisawa S (2003) Struct Chem 14:85-95

24. Ide-Ektessabi A, Fujisawa S, Yoshida S (2002) J Appl Phys 91:1613-1617

25. Ide-Ektessabi A, Rabionet M (2005) Anal Sci 21:885-892

26. Miller LM, Wang Q, Telivala TP, Smith RJ, Lanzirotti A, Miklossy J (2006) J Struct Biol 155:30-37

27. Geraki K, Farquharson MJ, Bradley DA (2004) Phys Med Biol 49:99-110

28. Geraki K, Farquharson MJ, Bradley DA (2002) Phys Med Biol 47:2327-2339

29. Ide-Ektessabi AFS, Sugimura K, Kitamura Y, Gotoh A (2002) X-Ray Spectrom 31:7-11

30. Carvalhoa ML, Magalhãesa T, Beckerb M, von Bohlen A (2007) Spectrochem Acta B 62:1004-1011

31. Rechner AC (ed) (2002) Methods of multivariate analysis. Wiley, Chichester

32. Drake EN, Sky-Peck HH (1989) Cancer Res 49:4210-4215

33. Paone G, De Angelis G, Munno R, Pallotta G, Bigioni D, Saltini C, Bisetti A, Ameglio F (1995) Eur Respir J 8:1136-1140

34. Marchevsky AM, Tsou JA, Laird-Offringa IA (2004) J Mol Diagn 6:28-36

35. Opstad KS, Ladroue C, Bell BA, Griffiths JR, Howe FA (2007) NMR Biomed 20:763-770

36. Krafft C, Sobottka SB, Geiger KD, Schackert G, Salzer R (2007) Anal Bioanal Chem 387:1669-1677

37. Falzon G, Pearson S, Murison R, Hall C, Siu K, Round A, Schültke E, Kaye AH, Lewis R (2007) Phys Med Biol 52:6543-6553

38. Louis DN, Ohgaki H, Wiestler OD, Cavenee WK (eds) (2007) WHO classification of tumours of the central nervous system. IARC, Lyon

39. Falkenberg G, Rickers K (2002) HASYLAB annual report, HASYLAB, Hamburg, pp 88-95. http://hasyweb.desy.de/science/ annual_reports/2002_report/index.html

40. http://www.iaea.org/OurWork/ST/NA/NAAL/pci/ins/xrf/downloads/ QXAS_Manual.pdf. Accessed 28 Jun 2011

41. Carroll JD, Green PE, Chaturvedi A (eds) (1997) Mathematical tools for applied multivariate analysis. Academic Press, New York

42. StatSoft (2005) STATISTICA 7.1. StatSoft, Tulsa

43. Munita CS, Barroso LP, Oliveira PMS (2006) J Radioanal Nucl Chem 269:335-338

44. Adamek D (2005) Folia Neuropathol 43:191-192

45. Giannini C, Burger PC, Berkey BA, Cairncross JG, Jenkins RB, Mehta M, Curran WJ, Aldape K (2008) Brain Pathol 18:360-369

46. Burger PC, Scheithauer BW (2008) In: Silverberg SG, Sobin LH (eds) Tumors of the central nervous system. ARP Press, Washington, pp 33-208 